\title{
Unmanned Aerial Vehicle Failure Modes Algorithm Modeling
}

\author{
P. Getsov, D. Yordanov, S. Zabunov
}

\begin{abstract}
The current article studies various predictable failure trajectories, observed during control signals loss scenarios. Using modeling techniques, certain failure mode algorithms are verified. The examined instances of failures are tested in the line-of-sight area. Certain autopilot failure regimes are studied and the resulting trajectories of airplane uncontrolled flight are scrutinized for determining failure resistant plane characteristics.
\end{abstract}

Keywords: flight control, autopilot, safety.

\section{INTRODUCTION}

The unmanned aerial vehicle (UAV) airplanes are piloted from a ground control station (GCS) by an operator or they are controlled by an onboard autopilot system. If the airplane does not receive commands from the GCS and at the same time the autopilot is malfunctioning, the plane will enter an emergency situation and the flight will be uncontrolled. On the other hand, if the autopilot is working upon loss of ground control then the former should enter failure mode and follow a predefined algorithm of flight. Using modeling techniques one could predict the most probable behavior of the flying apparatus. In all cases, risk exists of catastrophic termination of the flight process, because not all flight parameters are predictable. Further in this article, the autopilot unit dealing with failure modes shall be called safety unit. Fig. 1 and fig. 2 show a particular approach, modeled using the software application "Simulink".

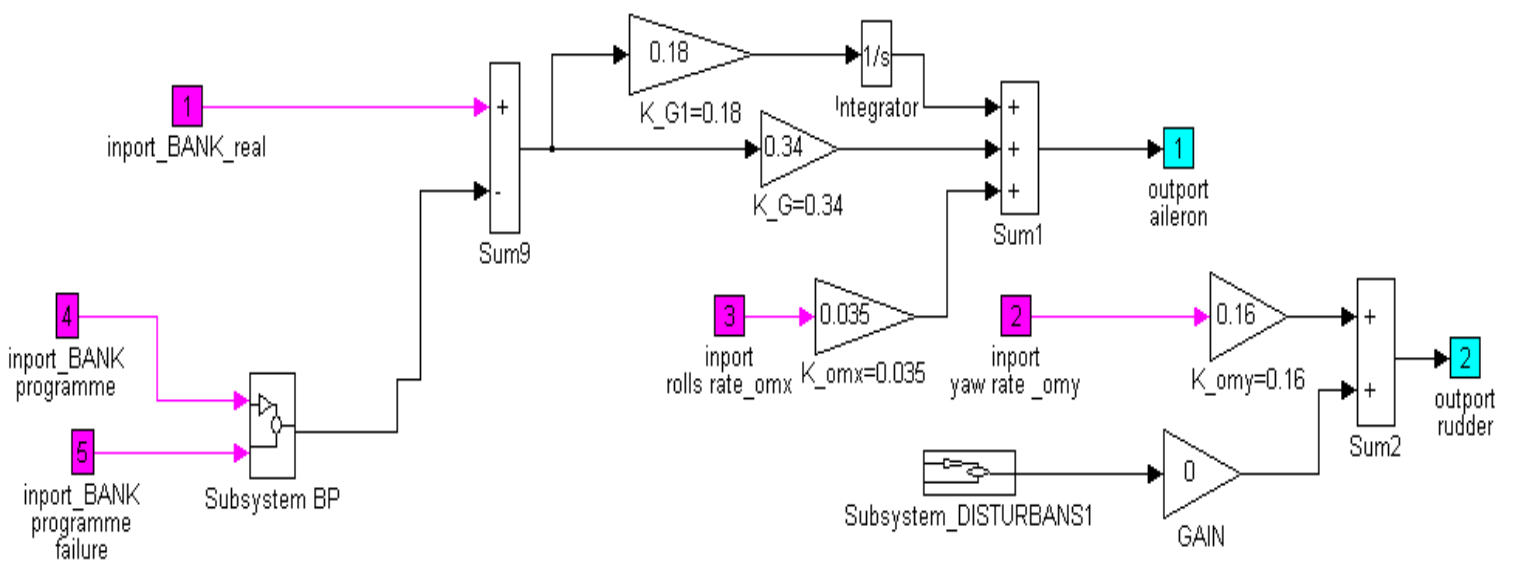

Fig. 1. Safety unit (Subsystem BP) controls the roll motion of the airplane

II. MODELING GENERAL FAILURE MODE

During flight the absolute value of the sum of plane altitude in meters and the roll angle in degrees is always greater than zero. This value is taken as an artificial control signal and it is sufficient as an indicator for lost control input (fig. 1).

A simple algorithm for emergency control is to enter the plane in a spiral trajectory with 15 degrees roll angle (fig. 2 and fig. 3). Such a flight trajectory would guarantee enough time for the operator to restore the control signals if possible. If the control signals are not restored the plane will land causing minor or no damage in the general case. 


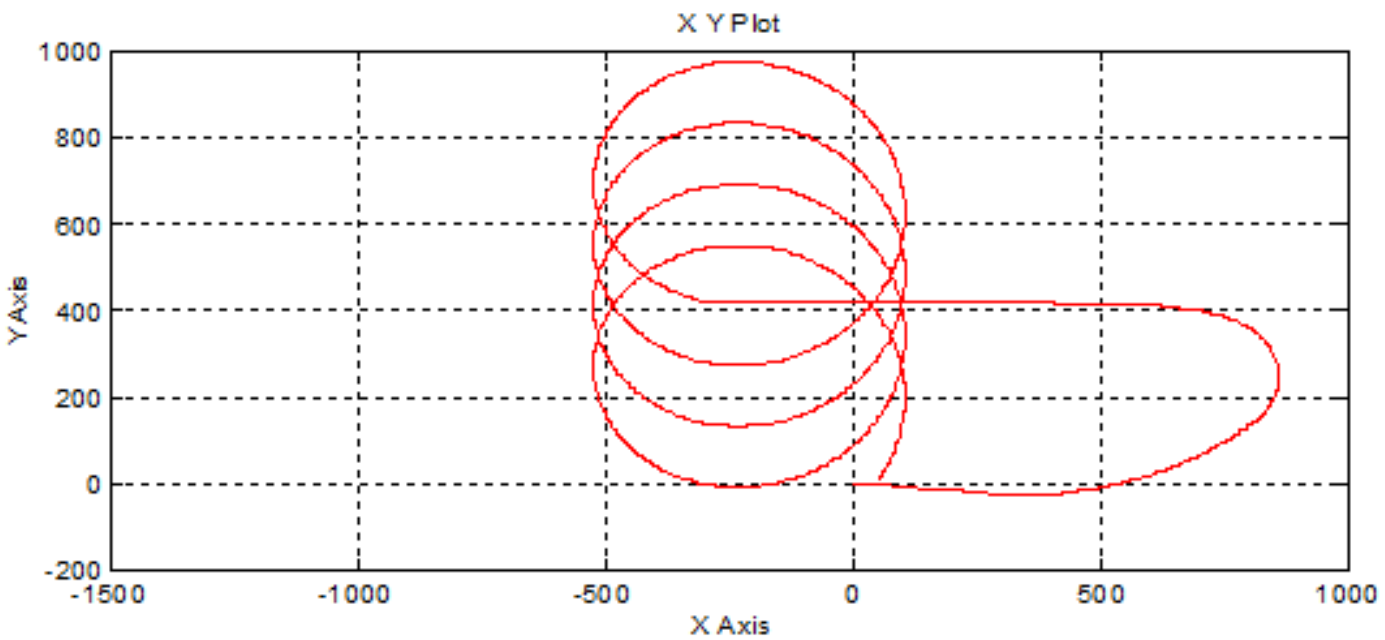

Fig. 2. Horizontal projection of the trajectory after control signal loss

The spiral shifting in failure mode (fig. 2) is due to modeled influence of side wind.

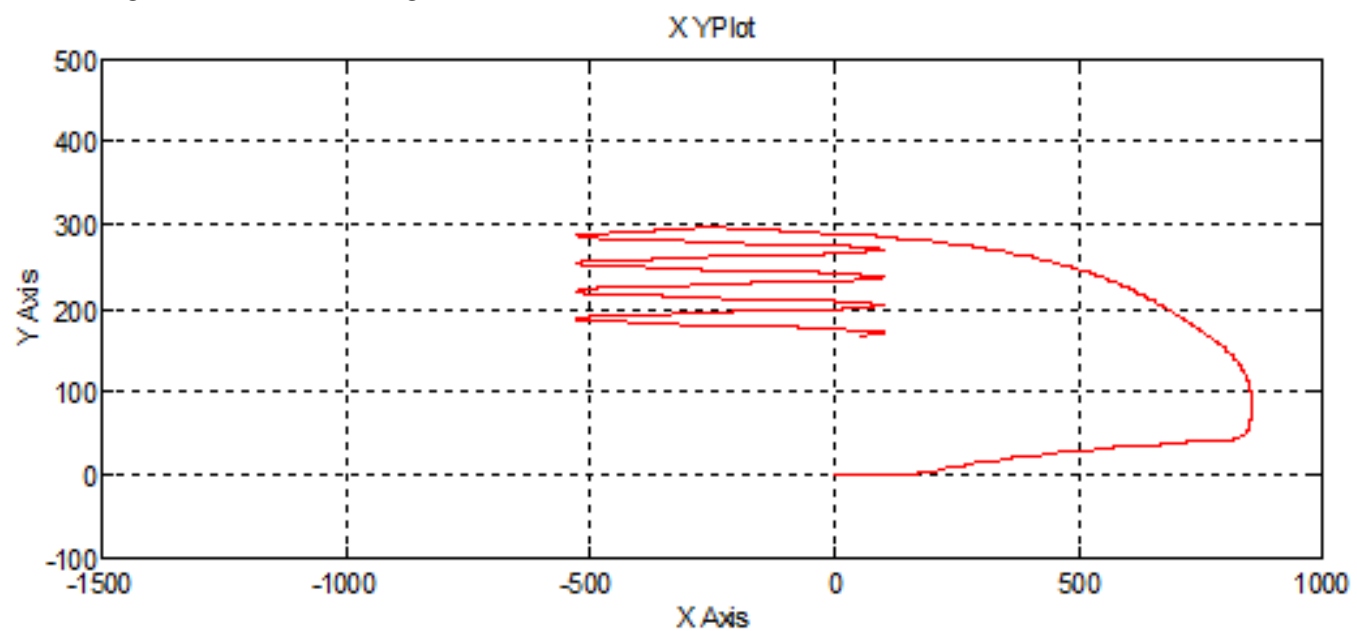

Fig. 3. Vertical projection of the trajectory after loss of control signals

Other algorithms are possible as well. For example, more appropriate may prove to be an altitude gaining spiral instead of descending spiral.

Fig. 2 and fig. 3 demonstrate emergency flight after signal loss. The signal loss is modeled to take place 80 seconds after takeoff. In normal flight, the airplane is performing typical automatic flight around the runway in the zone of line-of-sight using left turns. The presence of side wind is assumed. In failure mode the airplane stars to descend slowly in a spiral trajectory. The normal circular flight is shown on fig. 4 and is performed with course adjustments by the autopilot program. Takeoff is in manual mode till the $30^{\text {th }}$ second of the flight.
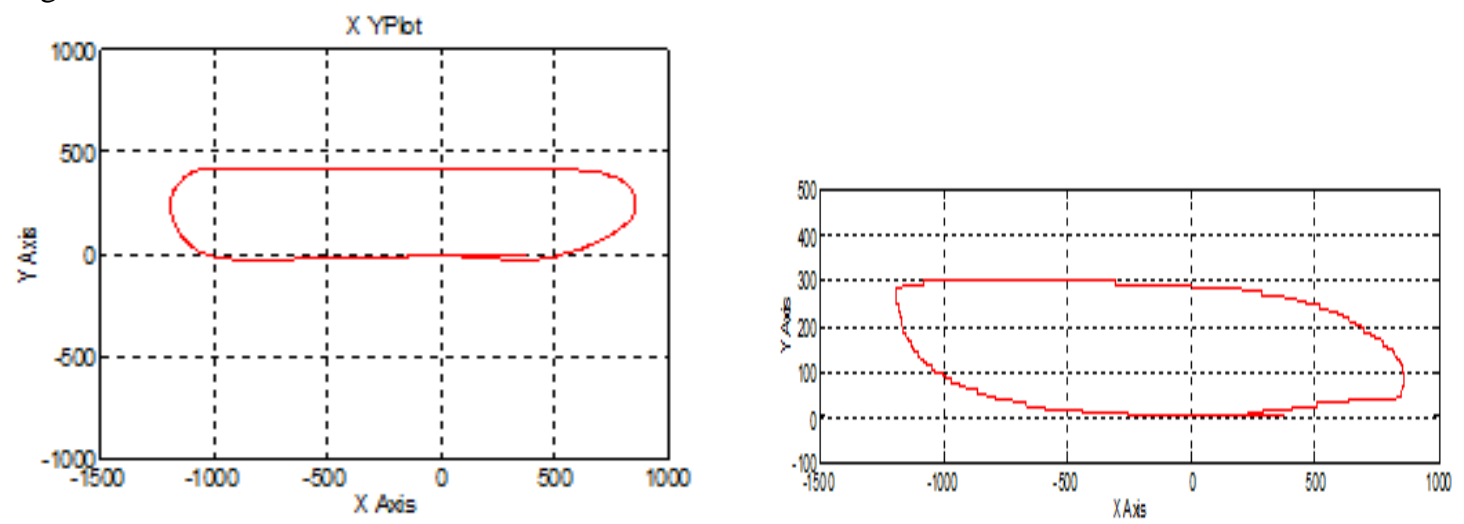

Fig. 4. Flight course (left) and flight profile (right) 


\section{MODELING DOUBLE CONTROL SIGNALS FAILURE}

The simultaneous control signals failure and autopilot failure is a very improbable situation. Its modeling is interesting because it happens seldom and generally leads to a crash. The catastrophic or noncatastrophic outcome of such a failure depends mainly on the ability of the plane to withstand spin motion stall. Figures 5, 6 and 7 disclose the results of a modeled complex emergency situation after two consecutive passive failures - failure of the radio control signals and failure of control signals to servo units. If the airplane is prone to spin stall a parachute system should be engaged, because the descent during spin stall is very steep.

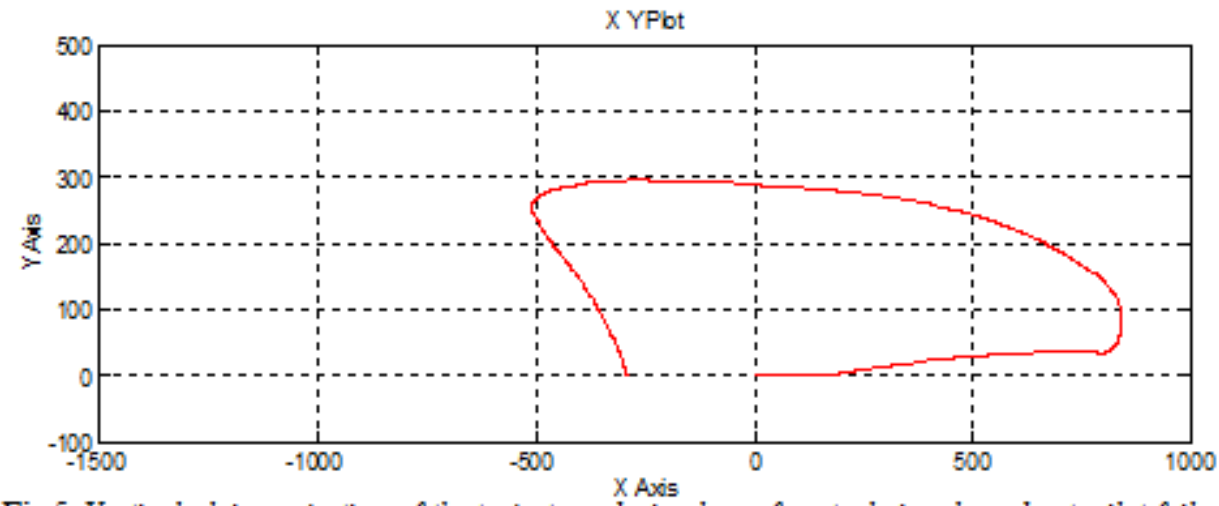

Fig.5. Vertical plain projection of the trajectory during loss of control signals and autopilot failure

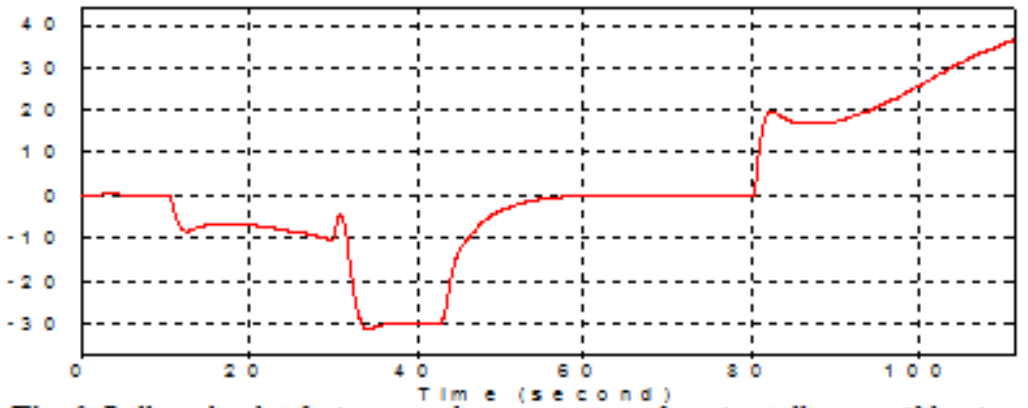

Fig. 6. Roll angle plot during complex emergency of a spin stall susceptible aircraft

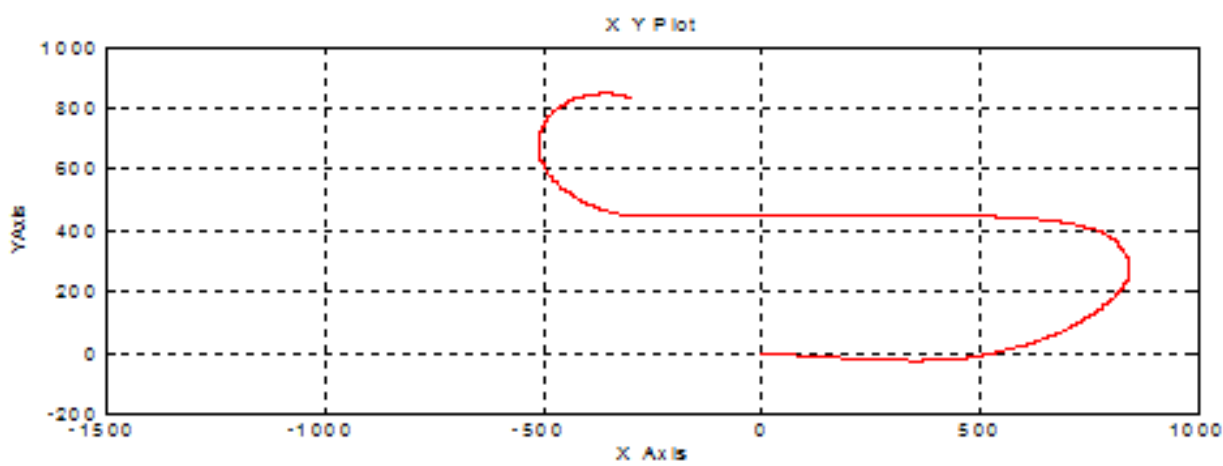

Fig. 7. Horizontal plain projection of the trajectory during loss of control signals and autopilot failure of a spin stall susceptible aircraft

If the plane is spin resistant, the emergency situation is non-fatal in the general case. The behavior of such a plane during full failure is shown on figures 8, 9 and 10 . 


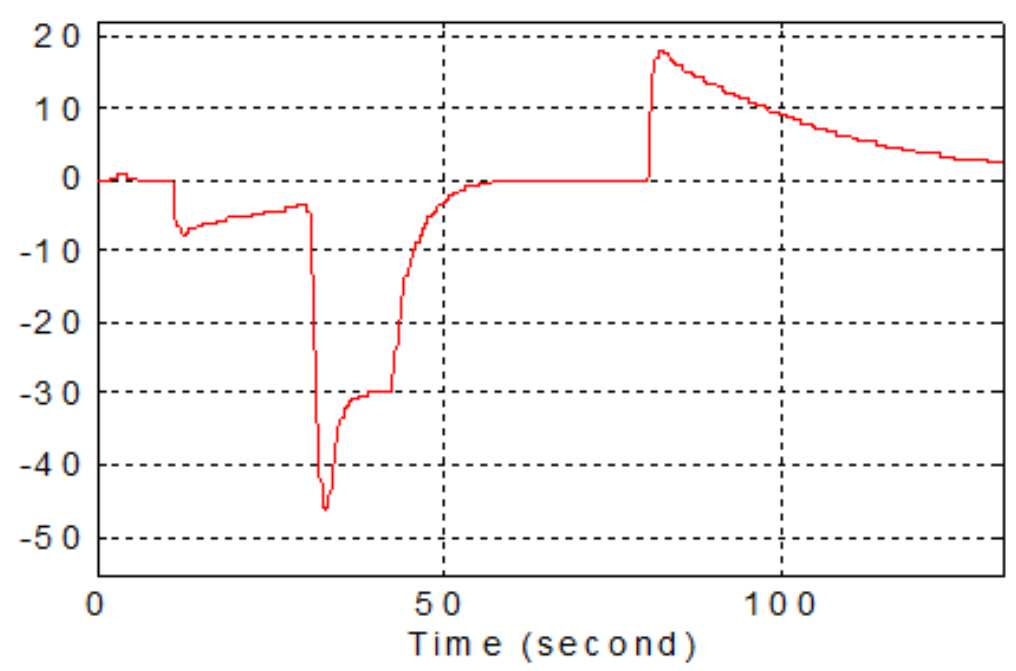

Fig. 8. Roll angle plot during emergency situation after loss of control signals in a spin resistant airplane

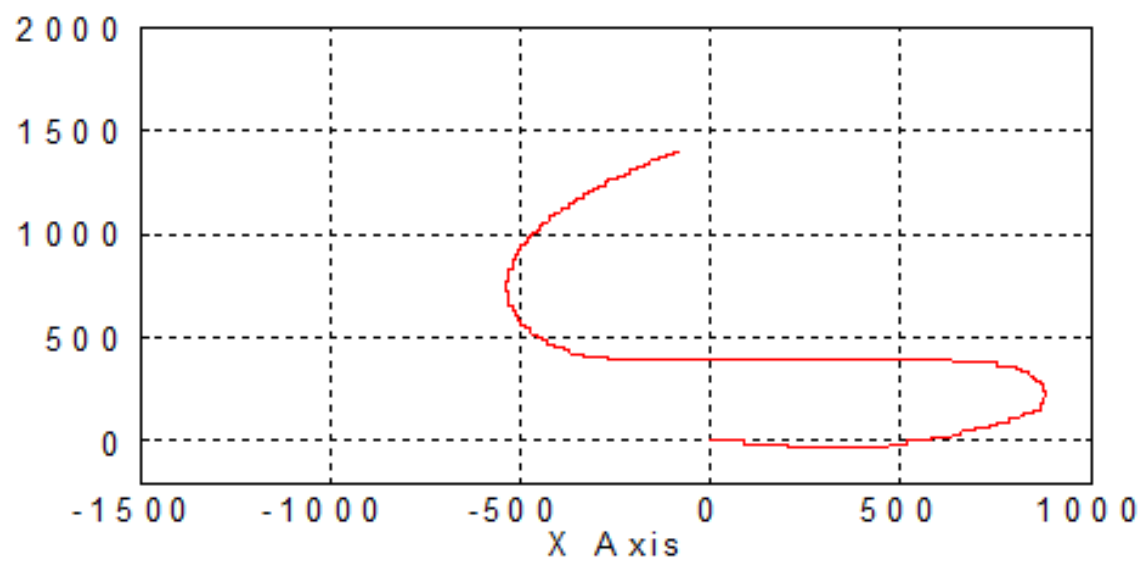

Fig.9. Horizontal projection of emergency trajectory in a spin resistant airplane

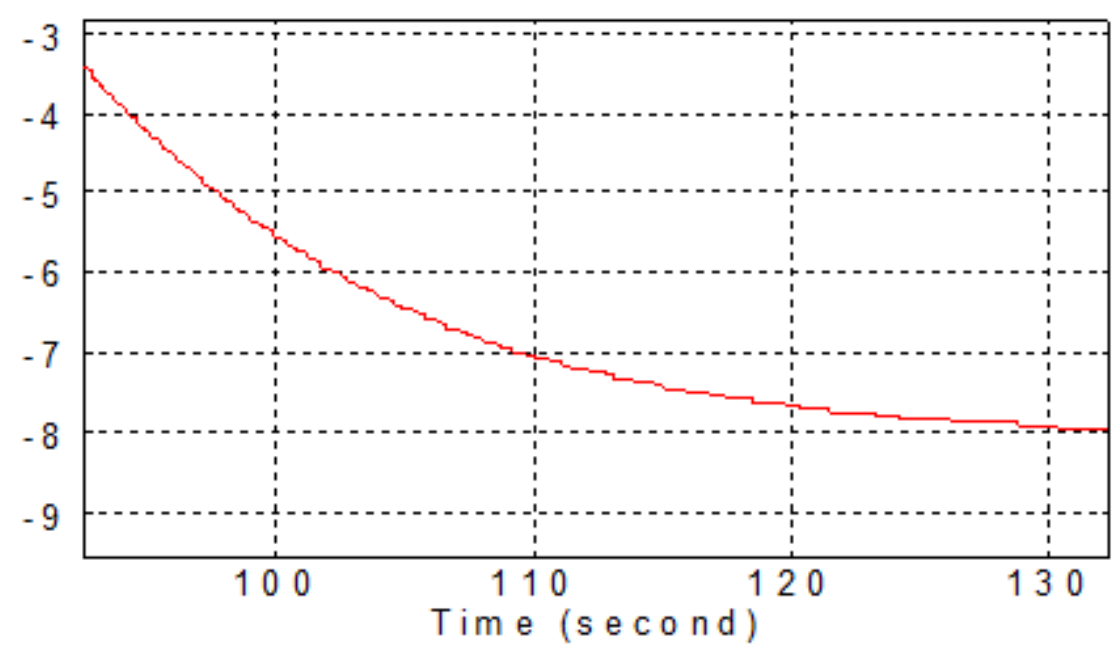

Fig. 10. Velocity plot before landing on ground in a spin resistant airplane 
IV.

\section{CONCLUSIONS}

Simulink modeling offers a simulation and analysis means for airplane flight in emergency situations such as control signals loss or autopilot failure.

Spin stall susceptibility is typical for some aircraft but under working and normal flight controls this quality is imperceptible even on piloted airplanes. The spin stall develops slowly and autopilot or pilot has time to counteract the spin development. The spin susceptibility is non-desirable in unmanned aircraft, because the consequences in an emergency situation are unfavorable for all objects participating in the emergency situation.

\section{REFERENCES}

[1] Mihalev I.A., B.N. Okoemov, I.G. Pavlina, M.S. Chikulaev, N.M. Eidinov. Automatic airplane control systems - methods for analysis and synthesis, "Mashinostroenie" publ., Moscow 1971 